\title{
Si tú escucharas lo que yo veo: la educación básica para sordos usuarios de la LSM
}

\author{
MIROSLAVA CRUZ-ALDRETE \\ Institución: Facultad de Humanidades, Universidad Autónoma del Estado de Morelos, México \\ MIGUEL ÁNGEL VILLA RODRÍGUEZ \\ Institución: Facultad de Estudios Superiores Zaragoza, UNAM, México.
}

\section{Introducción}

México vive una gran crisis en la educación de sus niños y jóvenes. Si bien, el Estado mexicano ha mandatado el derecho a la educación para todos sus ciudadanos, y promulgado que la educación básica sea obligatoria desde pre escolar hasta secundaria, es decir, doce años de escolaridad, esta disposición no resuelve el evidente rezago educativo que de manera general afecta a los diferentes grupos étnicos que conforman nuestra nación, entre ellos, la comunidad sorda.

En México, la comunidad sorda al igual que otros grupos lingüisticos minoritarios ha padecido por siglos la discriminación hacia su lengua y cultura que se traduce en el ámbito educativo en una carente o ineficaz instrumentación de políticas educativas y lingǘsticas que reconozca, entre otras cosas, la importancia del uso de su propia lengua la - lengua de señas - para la enseñanza. Pues si bien, hoy en día hay un reconocimiento de la Lengua de Señas Mexicana (LSM) como parte del patrimonio lingǘrstico y cultural de nuestra nación, en los hechos un gran sector de la población aún considera que el ideal educativo para el sordo es la oralización. Esta idea se traduce en la asimilación de los sordos a la cultura oyente y considerar el español como la única lengua de transmisión de la información, de enseñanza y de conocimiento.

En 1994, el movimiento zapatista de liberación nacional enfrentó a la comunidad mexicana a reconocer que la mayoría de los grupos excluidos de los servicios de salud y educativos tenían una condición en común, eran hablantes de lenguas indígenas. Esta situación no excluye a la comunidad sorda, usuaria de una lengua minoritaria, con una cultura, tradición e historia propias. El lema zapatista "Nunca más un México sin nosotros" fue compartido por todos aquellos que han sido marginados por no tener la lengua de la mayoría, en este caso, el español.

Hacemos hincapié en el hecho de que las comunidades sordas o las comunidades indígenas que tienen como lengua materna una lengua distinta a la lengua oral dominante ven limitadas sus oportunidades educativas. Hoy en día en México acceder a la educación en el nivel medio superior y superior, fundamentalmente, requiere que el alumno sea competente en la lengua oral dominante, en nuestro caso, el español.

Desde finales del siglo XX, en nuestro país, se ha venido instrumentando la propuesta de un modelo educativo bilingüe bicultural para la comunidad sorda. Dicho modelo ha sido adoptado y promovido tanto

Revista Iberoamericana de Educación / Revista Ibero-americana de Educação

ISSN: 1681-5653

n. ${ }^{\circ} 63 / 2-15 / 11 / 13$

Organizaciōn de Estados Iberoamericanos para la Educación, la Ciencia y la Cultura (OEI-CAEU)

Organização dos Estados Ibero-americanos para a Educação, a Ciência e a Cultura (OEI-CAEU) 
por el sector educativo público como privado. El modelo educativo bilingüe bicultural reconoce en principio la importancia de adquirir la LSM como primera lengua. Aunado a ello, implica la participación del maestro sordo - señante nativo de esta lengua - en la escuela como modelo lingüístico, y la necesidad de que haya maestros oyentes competentes en LSM, y no menos importante formados para la enseñanza del español en forma escrita como segunda lengua.

Ahora bien, estos elementos sólo hacen referencia de manera explícita el aspecto lingüístico, pero qué pasa con la cultura del sordo y del oyente que entran en contacto en la escuela. Desafortunadamente, hemos observado que este elemento es el gran ausente en la implementación del Modelo bilingüe bicultural para el sordo. Una perspectiva efectivamente bicultural resulta más complejo que la sola conjunción del maestro sordo y del maestro oyente en el aula, así como la mera inserción de la LSM como medio de enseñanza.

Así, a más de una década del desarrollo de este modelo en México, surgen preguntas de distinto indole sobre el estado actual del mismo. Estas interrogantes se relacionan por un lado con la población sorda, en el seguimiento de las generaciones que han sido formadas bajo este modelo y; sobre cuál ha sido el papel de la comunidad sorda en este enfoque educativo. Por el otro lado con la adquisición y enseñanza de la LSM y del español, es decir, sobre las cuestiones lingüisticas que se relacionan con la enseñanza del español en su forma escrita; y sobre la adquisición de la LSM como primera lengua para los sordos hijos de padres oyentes, y como segunda lengua para los oyentes. Algunas más se vinculan con la formación de recursos humanos: el perfil del maestro sordo y del maestro oyente. Así como la aparición en el escenario educativo del intérprete en lengua de señas (ILS) en el salón de clase, principalmente su incorporación en el nivel de secundaria y medio superior.

Sin duda, el 2012 reclama con urgencia la evaluación del modelo bilingüe bicultural. Este modelo se ha implementado por un lado, en los centros de atención múltiple (CAM) y, por el otro, sobre las experiencias incipientes en las escuelas a las cuales asisten en su mayoría alumnos oyentes. Por tanto, asumimos la postura de Margarita Zorrilla (2009) para quien la evaluación de la escuela es un reto ineludible, además de que debe considerarse como el punto de partida y de llegada de toda política educativa; $y$, de Elena Martín (2010), quien coloca en el centro de la discusión la importancia de evaluar lo que ocurre en la escuela para identificar y analizar el actuar de todos los que conformamos la comunidad educativa. Asimismo, remarca la necesidad de ponderar el contexto sociocultural donde se enmarca nuestra labor.

Con base en lo anterior, a partir del intercambio que hemos sostenido con distintos centros educativos de (Tijuana, Cuernavaca, Guadalajara, Distrito Federal, y Puebla) consideramos que no solo no hemos evaluado la parte operativa del Modelo Educativo Bilingüe Bicultural (infraestructura y formación de recursos humanos), sino que tampoco hemos revisado el marco teórico que lo sustenta. Resulta alarmante que no compartamos quienes implementamos este modelo el mismo cuerpo de conocimientos sobre el mismo. Observamos que cada centro escolar lo ha ejecutado desde sus creencias y con algunos elementos teóricos que se les han dado desde la Secretaría de Educación Pública, o por la capacitación que cada escuela ha buscado con su propia iniciativa. Si bien reconocemos que en un primer momento fue imperioso implementar el bilingüismo con las condiciones que se tenía (sin modelos sordos lingüísticos, maestros poco competentes en la LSM, escaso conocimiento sobre la postura antropológica ante la sordera, etcéteral, no podemos evadir la responsabilidad de evaluar a más de una década de implementado el modelo cuáles han sido sus alcances y limitaciones. Consideramos que no hemos discutido con la seriedad que se merece 
la eficacia de un modelo en el cual coexisten dos lenguas de distinta modalidad, pero, que además hemos obviado que están implicadas dos culturas.

Por otra parte, desde nuestra perspectiva, toda propuesta bilingüe bicultural o intercultural es el camino hacia la educación inclusiva y al logro de una cobertura educativa para la comunidad sorda, en el cual se garantice de manera efectiva su permanencia en la escuela al menos por doce años leducación inicial, primaria y secundaria). No podemos seguir desperdiciando como pueblo el potencial de la juventud sorda y perpetuando la pobreza y la discriminación hacia esa comunidad (Cruz-Aldrete 2009).

Un modelo bilingüe bicultural o intercultural promueve la convivencia de personas que provienen de grupos sociales con culturas, historias, costumbres y lenguas diferentes a las de la mayoría. Procura que todos los miembros de la comunidad educativa mantengan relaciones de igualdad y promueve el respeto a la diversidad. Asimismo, tiene como objetivo responder a las necesidades de todos y cada uno de los alumnos considerando la diversidad de lenguas y capacidades, asegurando el desarrollo armonioso e integral de toda la comunidad educativa (v. Unamuno 2003). De ahí la urgencia de revisar la ejecución de este modelo dirigido hacia la comunidad sorda, pues su evaluación nos permitiría saber si se ha alcanzado los objetivos propuestos.

La intención de este trabajo es comentar la sinergia que hemos experimentado entre los agentes de los distintos proyectos de educación bilingüe y bicultural que se realizan en Morelos, Guadalajara, Puebla, Tijuana y Distrito Federal. Considerando por un lado, la enseñanza en un escenario educativo como es el Centro de Atención Múltiple, y por el otro, la escuela regular ante la inclusión del alumnado sordo usuario de una lengua minoritaria, la LSM. Todos los participantes hemos puesto de manifiesto la necesidad de hacer un análisis reflexivo sobre el estado actual del Modelo Educativo Bilingüe y Bicultural para el sordo (MEBB) en nuestro país, así como la urgencia de establecer los medios para que las escuelas en donde se esté desarrollando se realice su evaluación, procurando que ésta no se convierta en un mero acto demagógico cuyo resultado no impacte en la construcción de una respuesta educativa para el sordo.

\section{La respuesta educativa ante la diversidad lingüística y cultural}

La documentación lingüística de México nos revela la existencia de alrededor de 11 familias de lenguas orales indígenas (68 agrupaciones lingüísticas) que coexisten con dos lenguas de señas hasta el momento documentadas, la Lengua de Señas Mexicana, empleada en toda la República Mexicana, y la Lengua de Señas Maya que se utiliza en la península de Yucatán. Si bien la lengua oral dominante utilizada por la mayor parte de la población es el español, el reporte de INEGI (2005) nos menciona que aproximadamente seis millones de personas de cinco años y más hablan alguna lengua indígena. Y de ese total de población 720 mil no hablan español.

Observamos que aun cuando en el discurso oficial continuamente se afirma que los sordos conforman comunidades lingüisticas minoritarias, en los hechos, a la persona sorda se le define por su "discapacidad" y no por el uso de una lengua con diferente modalidad. Si bien desde el 2005 se hizo un reconocimiento jurídico de la LSM considerándola parte del patrimonio lingüístico de la nación mexicana (Diario Oficial de la Federación 10 de junio de 2005), con lo cual se considera a lo sordos depositarios de una lengua y de una cultura, hoy en día los sordos siguen siendo vistos desde la perspectiva de la anormalidad. 
Desde nuestra óptica, no negamos el déficit auditivo que dificulta la adquisición de la lengua oral, pero sí ponderamos el derecho de la persona sorda de adquirir su propia lengua natural, la lengua de señas, la LSM.

Al respecto, si hiciéramos un breve recorrido histórico sobre la educación del sordo reconoceríamos que el foco de atención ha sido el aprendizaje de la lengua oral. Esto ha traído como consecuencia que el estatus de la lengua señas se haya mantenido durante mucho tiempo por debajo de la lengua oral dominante. Y que se considere a las señas como herramienta de enseñanza cuyo empleo permitirá la adquisición de la lengua de mayor prestigio, como discutiremos más adelante. Por tanto, no es de extrañar que la adopción de un modelo bilingüe bicultural para el sordo adolezca de varios elementos que ya son reconocidos y empleados en el ámbito de la educación de los pueblos originarios de nuestro país, sí su lengua sigue siendo en muchos sentidos desconocida y desprestigiada.

Un modelo bilingüe bicultural para el sordo debe propiciar el respeto por ambas lenguas LSMespañol, y por ambas culturas, sorda y oyente. Además, debe asegurar que los educandos sean competentes en ambas lenguas. Hasta el momento lo que hemos observado es una reducción del modelo, pues en algunas escuelas consiste en la sola inserción de la Lengua de Señas Mexicana en el aula, en la incorporación del maestro sordo como modelo lingüístico, pero no hay un trabajo de reflexión metalingüística de la LSM ni del español en su forma escrita, actividad fundamental que se requiere para hablar de un bilingüismo (Massone, Simon y Druetta 2003; Cruz-Aldrete y Cruz Cruz 2012).

Como es sabido, las líneas directrices para la enseñanza del sordo son definidas por el área de educación especial de la Secretaría de Educación Pública (SEP), y coordinadas por la Dirección de Educación Especial de cada uno de los estados que conforman la República Mexicana. Así, como por otras instancias emanadas de la SEP, por ejemplo, el Programa de Fortalecimiento de la Educación Especial y de la Integración Educativa, cabe destacar que en este programa se desarrollan documentos de trabajo para marcar líneas de acción en los Centros de Atención Múltiple (CAM), antes Escuelas de Educación Especial. Entre estos documentos de trabajo se encuentra la propuesta de un modelo bilingüe bicultural, y la inclusión de los niños sordos en las escuelas regulares (SEP 2010).

Bien, la política educativa para el sordo con un enfoque bilingüe se presenta de manera sucinta en El Diario oficial de la Federación del 10 de junio de 2005 en la Ley General de Personas con Discapacidad. En dicha ley se reconoce a la Lengua de Señas Mexicana (LSM) como una lengua nacional. Este reconocimiento implicó cambios en materia educativa, como podemos observar de manera general en lo dictaminado en el Capítulo III, Artículo 10, fracciones VIII, X, XI, XII y XIII concerniente a la educación en la cual se enfatiza el derecho de la población sorda a la educación obligatoria y bilingüe (LSM-español); la utilización de la LSM en el Sistema Educativo Nacional; la implementación de programas de formación certificación de intérpretes; impulsar el desarrollo y uso de la lengua escrita; el impulso de programas de investigación, preservación y desarrollo de la lengua de señas (v. revisión del 2008).

Como se puede observar, la ley reconoce el derecho de los sordos para recibir educación en su propia lengua. La interpretación del Artículo 10, con las fracciones que hemos presentado nos conduciría a la implementación de un modelo bilingüe. No obstante, no se han generado las condiciones necesarias para atender a la población sorda desde esta nueva perspectiva. A más de un lustro de la publicación de esta ley, 
todavía no se ha elevado una propuesta que coordine las acciones para formar maestros oyentes que sean competentes en LSM, maestros sordos que enseñen la LSM, y la preparación de intérpretes educativos.

Sin embargo, aun cuando estas disposiciones legales son recientes, destacamos el hecho de que la sociedad va más adelantada en la implementación de estrategias, y en la búsqueda de soluciones ante la problemática que se le presenta. En este sentido antes de que tuviera un sustento jurídico la educación bilingüe para el sordo, en algunas escuelas privadas y en algunos CAM ya se había puesto en marcha una propuesta bilingüe, pero, sin líneas directrices comunes. Si bien, esto constituyó un gran adelanto también trajo sus consecuencias, pues la práctica de este modelo en cada centro escolar dependió principalmente de la preparación de los recursos humanos con los que contaba, de su historia como escuela, así como de su propia reflexión sobre lo que suponía el desarrollo de una educación en donde coexistieran lenguas de distinta modalidad y con una gramática diferente. Y desafortunadamente, en algunos casos se siguieron perpetuando algunos falsos mitos sobre las lenguas de señas en cuanto a su gramática y calco con respecto al español.

Aunado a lo anterior resultaba menos asequible abordar la cultura sorda en la escuela. Este aspecto sigue siendo el más complejo, pues hoy en día al preguntar a los distintos involucrados en la ejecución de este modelo sobre qué es la cultura del sordo, no obtenemos respuesta.

Consideramos relevante que en la implementación de un modelo educativo bilingüe bicultural para el sordo se propicie que desde temprana edad los niños formen parte de su comunidad sorda. Es fundamental el intercambio generacional entre los adultos sordos y los niños sordos para la construcción de la identidad de sus miembros. Por lo que se refiere a la función de la escuela en esta construcción no debemos olvidar el papel de la Escuela Nacional de Sordomudos (ENS), pues no solo fue fundamental por la génesis de la LSM sino por la formación de una comunidad, por la transmisión de la cultura y de las tradiciones al interior de este grupo social (v. Jullian-Montañés 2001). Asimismo, porque puso de manifiesto la importancia de las relaciones entre los adultos y viejos de la comunidad sorda con las nuevas generaciones de sordos, y la importancia de su acompañamiento en la transición de la vida a infantil a la vida adulta. La necesidad de el mantenimiento de un padrinazgo en el contacto con la sociedad oyente. Coincidimos con Ramsey (2011) al mencionar que el cierre de la ENS trastocó la formación comunitaria del sordo, y por ello hoy resulta imperioso discutir que dentro de un modelo bilingüe bicultural es fundamental que en la escuela se construyan las relaciones comunitarias entre los niños, jóvenes, adultos y viejos sordos, propiciando su identificación como miembro de un grupo y una cultura.

\section{La evaluación del modelo educativo bilingüe bicultural}

Entre los trabajos pioneros sobre el Modelo Educativo Bilingüe (MEB) en México, encontramos el denominado Propuesta de educación bilingüe para el niño sordo en un Centro de Atención MúltiplelAcosta 2002) el cual fue elaborado bajo el auspicio de la Dirección de Educación Especial del Distrito Federal. En este trabajo ya se citaba la necesidad de la presencia de un modelo lingüístico sordo en el CAM y maestros competentes en LSM. A diez años de su puesta en marcha no contamos con documentos de libre acceso que reporten la evaluación de este modelo educativo. Ignoramos si se tiene un seguimiento de las generaciones que han egresado del CAM bajo este nuevo enfoque educativo. $Y$ no menos importante, 
desconocemos las reflexiones que como grupo de trabajo han tenido y que sin duda pueden servir de orientación a las escuelas que están en ese proceso de iniciar un MEB para el sordo.

Por otra parte, el equipo del MEB en el Distrito Federal ha elaborado materiales para el aprendizaje de la LSM, por ejemplo, el Diccionario Español Lengua de Señas Mexicana DIELSEME (2004); y la elaboración de un cuadernillo titulado Estrategias de trabajo para fortalecer la enseñanza y el aprendizaje del español escrito como segunda lengua en los alumnos sordos (SEP 2009) el cual según el equipo del MEB fue concebido como una herramienta que forma parte del Diccionario del Español-Lengua de Señas Mexicana II (DIELSEME II). Ambos materiales se distribuyeron en todos los Centros de Atención Múltiple del Distrito Federal.

Ahora bien, en la revisión del cuadernillo de estrategias para el aprendizaje del español escrito encontramos que carece de un sustento teórico suficiente, además de que no presentan los datos o la investigación realizada para hacer esta propuesta. En cuanto al diseño de las actividades que se presentan observamos que no se relaciona claramente con alguna propuesta de enseñanza de segundas lenguas. Asimismo, llama la atención que el desarrollo de las actividades que sugieren ya requiere de un grado de competencia en lengua escrita, pues, el principal problema que los profesores externan es la falta de un material adecuado para aquellos alumnos que inician con el aprendizaje de la lengua escrita, y demandan una respuesta a esta necesidad.

Aun cuando la educación del sordo está circunscrita al ámbito de educación especial, consideramos que es necesario recuperar la experiencia que se tiene desde el ámbito de la educación bilingüe intercultural dirigido a las comunidades indígenas para la elaboración de materiales, pues se requiere de unir esfuerzos con pedagogos, lingüistas y miembros de otras disciplinas para su creación. Hoy en día la educación requiere de sumar experiencias de distintos ámbitos y la enseñanza del sordo no debiera ser la excepción.

Basándonos en la observación y el trabajo que hemos venido realizando con centros educativos del DF, Morelos, Guadalajara, Puebla y Tijuana, de manera general podemos decir que los avances en el modelo educativo bilingüe bicultural para el sordo se han dado principalmente en el interés de los docentes en aprender LSM, en la incorporación de la figura del intérprete, y de la participación como agente educativo de un sordo nativo señante de la LSM. De la misma manera, podemos decir que comparten la misma problemática en cuanto a la disponibilidad de recursos humanos. Siguen siendo pocos los maestros sordos señantes laborando en los CAM, cuentan con un número limitado de maestros oyentes competentes en LSM, en contraste con una gran una mayoría de maestros oyentes que desconocen esta lengua, o bien, dicen conocerla, pero, la realidad es otra, no respetan la gramática de la LSM e incluso inventan señas. Y, más delicado aún resulta el hecho de que haya maestros que no han modificado su actitud hacia las lenguas de señas, evidenciando así una falta de respeto hacia su alumno sordo.

La falta de maestros sordos y de maestros oyentes competentes en LSM implica que la transmisión de la lengua no se esté dando de manera adecuada. Desafortunadamente hemos podido observar alumnos sordos que van adquiriendo la LSM utilizando señas que no son propias de esta lengua, así como produciendo construcciones sintácticas que presentan el orden de constituyentes del español y no el de la LSM. Si bien se ha puesto un énfasis en la enseñanza de la LSM como segunda lengua para abatir esta problemática general, desde la dirección de educación especial de la SEP de algunos estados, entre ellos Jalisco, Baja California, Morelos, Distrito Federal, impartiendo cursos y talleres dirigidos a maestros y a 
padres de familia, bajo la coordinación de maestros sordos nativos señantes de la LSM, de maestros oyentes competentes en esta lengua, y de intérpretes en LSM-español; no se ha hecho una evaluación sistemática sobre este trabajo.

Por tanto, habría que indagar cuál ha sido el resultado de ese trabajo: si los maestros han cambiado su práctica educativa y emplean la LSM para la enseñanza; si los padres de familia utilizan la LSM para comunicarse en casa; si se han relacionado con la comunidad sorda, si han cambiado la actitud que tienen hacia la LSM y hacia la condición de la sordera. También habría que discutir sobre la calidad de los materiales que se han realizado con este fin, por ejemplo, Las maravillas de la comunicación están en nuestras manos (Salazar-Romero \& Ibarra Mercado, 2009) o el Diccionario DIELSEME I (2004), recursos que se han repartido en los CAM y cuya creación, distribución y uso, ha sido con el objetivo de favorecer el aprendizaje de la LSM. Esta evaluación es una tarea pendiente que sin duda permitiría definir nuevos caminos que apuntalen el desarrollo del modelo bilingüe bicultural.

Otro punto importante a evaluar es la actitud de los niños hacia la LSM y el español. Los niños sordos (y oyentes) deben observar que la LSM es una lengua de prestigio al igual que el español, que se puede "decir todo" en LSM. Al respecto debemos destacar el trabajo realizado en el CAM Hellen Kéller de Tijuana, cuyo personal en conjunto con un colectivo de diseñadores (Fundación que transforma) han desarrollado cápsulas informativas con contenidos de distinto índole, por ejemplo, de ecología, donde los propios niños sordos (alumnos del CAM) en LSM transmiten esa información. Son cápsulas que se transmiten por el canal local de televisión, en donde una voz en off utilizando el español va narrando lo que los niños explican en LSM. Es un claro ejemplo de lo que se puede hacer entre sordos y oyentes, aunque desafortunadamente esta labor es casi desconocida por el resto de escuelas del país.

Otro ejemplo de colocar a la LSM más allá del aula de educación especial es el trabajo que se realiza en Guadalajara Jalisco, con el equipo encargado del Programa de Enseñanza Bilingüe (Lengua de Señas Mexicana-Españoll, el cual ha salido del ámbito de educación especial. Los maestros sordos participan activamente en la enseñanza de la LSM en la escuela regular, y en los servicios de salud. De esta manera contribuyen a la sensibilización de la comunidad oyente con respecto a la existencia de otras lenguas, como es la LSM, y el respeto a los sordos como personas hablantes de otra lengua.

Ahora bien, algunas organizaciones civiles y grupos de profesionales en distintas partes de nuestro país, han logrado que diversas preparatorias y universidades (públicas y privadas) tengan disposición para que alumnos sordos compartan sus aulas con alumnos oyentes. Estos grupos han generado sus propias estrategias de enseñanza, integración, y abriendo el camino para la inclusión. Por ejemplo, han incorporando en su planta docente a la figura del intérprete LSM-español-LSM. Sin embargo, este trabajo en su mayoría ha sido de manera intuitiva. Tal es el caso de la experiencia de la comunidad sorda y oyente en CONALEP (Colegio Nacional de Educación Profesional Técnica) estado de Morelos. Los profesores de esta institución se aventuran en el 2005 a vivir la experiencia de atender en un mismo grupo alumnos sordos y oyentes, sin un conocimiento previo sobre lo que implicaría tener un grupo con tales características. Pero, sí con una gran disposición para hacer esta labor. Los alumnos sordos comparten el aula y las clases con sus compañeros oyentes. Para ello se cuenta con un intérprete que está en el grupo de manera constante y en todas las actividades. 
En el 2005, CONALEP Morelos, plantel Temixco, abre sus puertas por primera vez a cuatro alumnos sordos, para que cursen la carrera de Alimentos y bebidas. Esta apertura a atender a los jóvenes sordos fue debido en gran parte a la iniciativa y participación de la Asociación educativa, cultural y recreativa para Sordos de Morelos A.C. Es la primera generación de alumnos sordos (2005 -2008) en concluir sus estudios a nivel medio superior en todo el Estado de Morelos.

En esta experiencia educativa los sordos usuarios de la LSM se enfrentan no solo a la exigencia de un nivel escolar superior, sino a otro medio con compañeros y maestros oyentes generalmente monolingües en español. Reconociendo que en algunos casos carecen de los conocimientos necesarios que le permitan establecer aprendizajes significativos en igualdad de condiciones con respecto a sus compañeros oyentes.

Es innegable que los sordos que quieren continuar sus estudios se enfrentan a varios retos. Sabemos por nuestra experiencia al ser docentes de alumnos sordos y oyentes, cómo la diferencia en su formación como estudiantes, y en sus competencias lingüísticas y comunicativas en su segunda lengua el español, los pone en desventaja; se dificulta el aprendizaje, es un gran esfuerzo el que hacen los alumnos sordos, el cual no se traduce en un éxito escolar ni en una adecuada formación académica.

Ahora bien, el acceso a la educación media superior y superior para el sordo introdujo por primera vez la figura del intérprete en el salón de clases como un agente educativo. Los alumnos sordos destacan el papel del intérprete a quien consideran como un elemento fundamental en su formación. Consideran que es alguien imprescindible para su aprendizaje y permanencia en la escuela. De ahí que desde su punto de vista consideran necesario que el intérprete cumpla además de su función principal, con la realización de tutorías dentro y fuera del aula. Y, de un acompañamiento/apoyo en la realización de algunos trámites administrativos que la escuela suele demandarles. Estos comentarios colocan al intérprete en un lugar relevante, y por ello es pertinente discutir cuál es el papel del intérprete en el aula. Hacer una evaluación de su perfil y la definición de las actividades que debe tener. Consideramos que la falta de claridad de su papel en el aula conduce a que hoy por hoy se le solicite funciones que no le corresponden. Si bien algunos ILS la han adoptado a partir de su labor diaria con el grupo de alumnos sordos, esto no significa que sea lo mejor para los alumnos, profesores y para el propio intérprete.

Aunado a lo anterior, cabe mencionar que en las escuelas donde se requiere intérpretes se enfrentan con el problema de la asignación de recursos para su contratación, pues es una figura que no forma parte de sus actuales organigramas. Por tanto, la mayoría de las veces son los padres de los alumnos sordos que tienen que pagar al intérprete, el cual además suele ser uno solo el que esté interpretando todas las asignaturas, un trabajo que resulta extenuante y del cual no se ha reconocido su importancia.

La función y formación del intérprete "educativo" requiere de un mayor análisis e investigación, que sobresale de los límites de este trabajo, no obstante queremos enunciar algunas preguntas que sirvan para iniciar una discusión al respecto: ¿cómo, dónde y con quién se forman los intérpretes en LSM? Y en cuánto a su papel en la educación nos interesa saber ¿quién regula su actividad como intérprete en el salón de clases? 


\section{Conclusiones y comentarios}

Ya para finalizar, queremos comentar que si bien, era necesario poner en marcha el modelo bilingüe aun con los pocos elementos con los que se contaba lteórico metodológicos, infraestructura y recursos humanos), ahora, en la segunda década del siglo XXI, no podemos rehusar a hacer una evaluación de lo que ha ocurrido en las escuela donde se desarrolla el Modelo bilingüe bicultural para el sordo. Compartir de manera abierta cuáles han sido sus alcances, cómo se ha articulado la escuela especial con la escuela regular, cuáles han sido sus obstáculos, y cuál ha sido el papel de la comunidad sorda en el ejercicio de su liderazgo para la planeación y ejecución de la educación que requiere.

Es indudable que una atención a la diversidad contribuye al enriquecimiento de cualquier sociedad. Sin embargo, el objetivo de una educación para todos, ha colocado peligrosamente al sordo en una situación escolar no planificada, atentando así a su derecho de recibir una educación con calidad y equidad. Es decir, no basta con esgrimir el argumento de que los sordos tienen las mismas oportunidades educativas si antes no se reflexiona y evalúan las condiciones en la cuales se lleva a cabo el proceso enseñanza aprendizaje, si no se conoce cuál es la red o el soporte con el que cuenta el alumno sordo, el alumno oyente, el profesor oyente, el intérprete, la familia, etcétera, elementos que han sido discutidos por autores como Domínguez (2009) quien destaca el desarrollo de redes de colaboración, apoyo y ayuda para la inclusión de los alumnos sordos.

Nos sumamos a la reflexión de Pilar Fernández Viader (2009) quien anota que una respuesta educativa que soslaye los aspectos diferenciales, distintivos de la sordera difícilmente puede proporcionar a los jóvenes sordos los recursos necesarios para su completo desarrollo cognitivo, lingüístico y social, y alcanzar, posteriormente, una formación cultural adecuada. La educación del sordo en nuestro país sigue siendo un problema que no hemos atendido con la seriedad que se merece.

Para finalizar queremos agradecer a la comunidad educativa de los distintos centros de educación especial pertenecientes a la Secretaría de Educación Pública (Tijuana, Guadalajara, Distrito Federal) y de CONALEP Morelos, el compartir su experiencia con una mirada crítica y propositiva. Así como por permitirnos ser parte de su formación y tarea educativa. Sea pues este un reconocimiento a su labor. Sin duda, el trabajo que desarrollan es un ejemplo del camino hacia la inclusión educativa, al respeto a la diversidad cultural y lingüistica de todos los grupos que conforman nuestra nación.

\section{Bibliografía}

ACOSTA, Lourdes (2002). Propuesta de Educación Bilingüe para el niño sordo en un Centro de Atención Múltiple del Distrito Federal. México: Dirección de Educación Especial.

ACOSTA Lourdes, CALVO Tere, MAYA Daniel, SANABRIA Edgar (2004). Diccionario Español-Lengua de Señas Mexicana (DIELSEME). México: Dirección de Educación Especial, SEP.

CRUZ-ALDRETE, Miroslava (2009). "Reflexiones sobre la Educación bilingüe Intercultural para el sordo en México". Revista Latinoamericana de Educación Inclusiva. 3(1): 133-145. Santiago de Chile: Universidad Central de Chile, RINACE, Red Iberoamericana de Investigación sobre Cambio y Mejora de la Escuela.

CRUZ-ALDRETE Miroslava \& CRUZ-CRUZ Johan (2012). "Expectativas familiares ante el modelo educativo inclusivo con jóvenes sordos del CONALEP del estado de Morelos, México". Revista Latinoamericana de Inclusión educativa. 6(1):143-152. Santiago de Chile: Universidad Central de Chile, RINACE, Red Iberoamericana de Investigación sobre Cambio y Mejora de la Escuela. 
DIARIO OFICIAL DE LA FEDERACIÓN (DOF). Ley General de las Personas con Discapacidad. México: 10 de junio de 2005. Última reforma publicada DOF el 01 de agosto de 2008. Disponible en: $<\mathrm{http}$ ://www.archivos.uait/legislación_univ2012/Leyes_federales_03/

Ley_General_de_las_Personas_con_Discapacidad.pdf [Consulta: junio de 2012].

DOMINNGUEZ, Ana Belén (2009). "Educación para la inclusión de alumnos sordos". Revista Latinoamericana de Educación Inclusiva. 3(1):45-61. Santiago de Chile: Universidad Central de Chile, RINACE, Red Iberoamericana de Investigación sobre Cambio y Mejora de la Escuela.

FERNÁNDEZ-VIADER, María del Pilar (2009). "Modelos actuales de educación bilingüe para los sordos en España". Rivista di psicolinguistica applicata. (VIII):257 -277. Pisa, Roma: Fabrizio Serra Editore.

JULLIAN-MONTAÑÉS, Christian (2001). Génesis de la comunidad silente en México. La Escuela Nacional de Sordomudos (1867 a 1886). [Tesis de licenciatura]. México: UNAM.

INEGI (2005). || Conteo de población y vivienda. México: Instituto Nacional de Estadística y Geografía.

MARTíN, Elena (2010). "Comentarios en el foro de evaluación". En OEl. Síntesis del debate de las Metas Educativas 2021. Madrid: OEl.

MASSONE María Ignacia, SIMÓN Marina, DRUETTA Juan Carlos. Arquitectura de la escuela de sordos. Libros en red, 2003. Disponible en: http://www.librosenred.com

RAMSEY, Claire L. (2011). The people who spell. The last students from the Mexican National School for the Deaf. Washington, DC: Gallaudet University Press.

SALAZAR-ROMERO Cristina \& IBARRA-MERCADO Adrián (2010). Las maravillas de la comunicación están en nuestras manos. Escucha mis manos y sabrás lo que digo. México: Gobierno de Jalisco. Secretaría de Educación.

SEP (2009). DIELSEME II. Diccionario Lengua de Señas Mexicana. Estrategias para fortalecer la enseñanza y el aprendizaje del español escrito como segunda lengua en los alumnos sordos. México: Dirección de Educación Especial, Secretaría de Educación Pública.

SEP (2010). Orientaciones desde el enfoque bilingüe para la atención educativa de alumnos sordos que cursan la educación básica. México: Secretaría de Educación Pública.

UNAMUNO, Virginia (2003). Lengua, escuela y diversidad sociocultural. Hacia una educación lingüística crítica. Barcelona: GRAÓ.

ZORRILLA, Margarita (2009). "Re-pensar la escuela como escenario del cambio educativo". En A. Marchesi, J.C Tedesco y C. Coll (Coords.) Calidad, equidad y reformas en la enseñanza. Madrid: OEl. 